\title{
Naked Dense Bodies Provoke Depression
}

\author{
Stefan Hallermann, ${ }^{1 \star}$ Robert J. Kittel, ${ }^{1,2,4 \star}$ Carolin Wichmann, ${ }^{2,3 \star}$ Annika Weyhersmüller, ${ }^{1}$ Wernher Fouquet, ${ }^{2,6}$ \\ Sara Mertel, ${ }^{2}$ David 0wald, ${ }^{2,3,6}$ Stefan Eimer, ${ }^{5}$ Harald Depner, ${ }^{3,6}$ Martin Schwärzel, ${ }^{3}$ Stephan J. Sigrist, ${ }^{2,3,6}$ \\ and Manfred Heckmann ${ }^{1,2,4}$ \\ ${ }^{1}$ Carl-Ludwig-Institute of Physiology, Medical Faculty, University of Leipzig, 04103 Leipzig, Germany, ${ }^{2}$ Rudolf Virchow Center, DFG Research Center for \\ Experimental Biomedicine, University of Würzburg, 97078 Würzburg, Germany, ${ }^{3}$ Institute for Biology/Genetics, Free University Berlin, 14195 Berlin, \\ Germany, ${ }^{4}$ Institute of Physiology, University of Würzburg, 97070 Würzburg, Germany, ${ }^{5}$ European Neuroscience Institute-Göttingen, Center for Molecular \\ Physiology of the Brain, Medical Faculty, University of Göttingen, 37077 Göttingen, Germany, and ${ }^{6}$ Cluster of Excellence NeuroCure, Charite, 10117 Berlin, \\ Germany
}

At presynaptic active zones (AZs), the frequently observed tethering of synaptic vesicles to an electron-dense cytomatrix represents a process of largely unknown functional significance. Here, we identified a hypomorphic allele, $b r p^{\text {nude }}$, lacking merely the last $1 \%$ of the C-terminal amino acids (17 of 1740) of the active zone protein Bruchpilot. In $b r p^{\text {nude }}$, electron-dense bodies were properly shaped, though entirely bare of synaptic vesicles. While basal glutamate release was unchanged, paired-pulse and sustained stimulation provoked depression. Furthermore, rapid recovery following sustained release was slowed. Our results causally link, with intramolecular precision, the tethering of vesicles at the $\mathrm{AZ}$ cytomatrix to synaptic depression.

\section{Introduction}

The rate at which sensory information can be transmitted across synapses is an important factor that limits rapid and precise information processing in the nervous system. The mechanisms that operate in the presynaptic terminals to release neurotransmitter at AZs at high repetition rates are currently intensely investigated (Atwood and Karunanithi, 2002; Zucker and Regehr, 2002; Kidokoro et al., 2004; Neher and Sakaba, 2008). AZs are decorated with electron-dense material of varying shape and size depending on synapse type and species (Zhai and Bellen, 2004).

Recently, the CAST/ERC-family member Bruchpilot (BRP), a coiled-coil rich protein of nearly $200 \mathrm{kDa}$, was identified via its localization to Drosophila AZs. BRP was shown to be crucial for cytomatrix formation, proper $\mathrm{Ca}^{2+}$ channel clustering within AZs, and efficient neurotransmitter release at the Drosophila neuromuscular junction (Kittel et al., 2006; Wagh et al., 2006). Furthermore, BRP is a direct component of the electron-dense cytomatrix (T-bar) and adopts an elongated conformation, with its $\mathrm{N}$ terminus ( $\mathrm{N}$-term) facing $\mathrm{Ca}^{2+}$ channels at the membrane and its C-term reaching into the cytoplasm (Fouquet et al., 2009).

Received May 15, 2010; revised Aug. 12, 2010; accepted Aug. 26, 2010.

This work was supported by grants from the Deutsche Forschungsgemeinschaft (DFG) to S.J.S. (Exc 257, SI849/ 2-1, TPA16/SFB 406, TP B23/SFB 581) and to M.H. (HE2621/4-2 and TP B27/SFB 581), and by formel. 1 grants to S.H. and R.J.K. from the Medical Faculty of the University of Leipzig. R.J.K. is supported by the DFG Emmy NoetherProgram (KI 1460/1-1). We acknowledge the VDRC for the cacophony RNAi line.

*S.H., R.J.K., and C.W. contributed equally to this work.

This article is freely available online through the J Neurosci Open Choice option.

Correspondence should be addressed to either of the following: Manfred Heckmann, Physiologisches Institut, Röntgenring 9,97070 Würzburg, Germany, E-mail: heckmann@uni-wuerzburg.de; or Stephan J.Sigrist, Institute for Biology/Genetics, Free University Berlin, Takustrasse 6, 14195 Berlin, Germany, E-mail: stephan.sigrist@fu-berlin.de.

DOI:10.1523/JNEUROSCI.2495-10.2010

Copyright $\odot 2010$ the authors $\quad 0270-6474 / 10 / 3014340-06 \$ 15.00 / 0$

\section{Materials and Methods}

Genetics. Larvae were raised at $25^{\circ} \mathrm{C}$ [cacophony (Cac) RNA interference (RNAi) larvae and their controls were raised at $29^{\circ} \mathrm{C}$ ] in bottles or on apple agar plates (for high-pressure freezing), and the following genotypes were used: Bruchpilot studies: $b r p^{\text {nude }}$ or $b r p^{1.3}$ or $b r p^{5.45}$ / df(2R)BSC29, ok6-GAL4 (Fouquet et al., 2009), controls: +/ok6-GAL4. Cacophony images: ok6-GAL4, UAS-Cac ${ }^{G F P} /+$ and $b r p^{\text {nude }} / d f(2 R) B S C 29$, ok6-GAL4; UAS-Cac ${ }^{G F P} /+$. Cacophony RNAi studies: UAS-Cac RNAi (Transformant ID 5551) (Dietzl et al., 2007)/+;elav-GAL4/+, control: elav-GAL4/+.

Stimulated emission depletion microscopy. Stimulated emission depletion (STED) microscopy experiments were performed essentially as described by Kittel et al. (2006), using the commercially available Leica TCS STED microscope (Leica Microsystems). Secondary antibodies were conjugated to the Atto $647 \mathrm{~N}$ dye (AttoTech). Atto $647 \mathrm{~N}$ fluorophores were excited at a wavelength of $635 \mathrm{~nm}$ and depleted at $750 \mathrm{~nm}$. Images were acquired using APD detectors within a range of $645-715 \mathrm{~nm}$. Image processing was performed using the Imspector software (Max-Planck Innovation) by applying a linear deconvolution at single STED slices.

To assess $\mathrm{Ca}^{2+}$ channel clustering, neuromuscular junctions were stained with M- $\alpha$-GFP (Invitrogen) and G- $\alpha$-M-Atto647N to visualize $\mathrm{Ca}^{2+}$ channels $\left(\mathrm{Cac}^{\mathrm{GFP}}\right)$. Additionally, serving as a reference, postsynaptic glutamate receptors were labeled with primary $\mathrm{Rb}-\alpha$-DGluRIID and secondary Sheep- $\alpha$-Rb-Cy3 (Invitrogen) antibodies and imaged via conventional confocal microscopy. From such images, AZs were selected that appeared planar to the optical slice (i.e., the AZ membrane is parallel to the focal slice). With Mathematica 5.0 (Wolfram Research), each channel (Cac ${ }^{\text {GFP }}$ and GluRIID) of each image of a planar AZ was automatically fitted with a two-dimensional Gaussian function. The peak of these Gaussians was used to align the Cac ${ }^{\mathrm{GFP}}$ signal either with itself or with the GluRIID signal for subsequent averaging. The average images were again fitted with two-dimensional Gaussian functions and both horizontal and vertical intensity profiles through the peak of the twodimensional Gaussian functions were averaged and fitted with onedimensional Gaussian functions (see Fig. 2 B). 
Electron microscopy. Electron microscopy and conventional embedding was performed as previously described (Fouquet et al., 2009) on late third-instar Drosophila larvae. The number of docked vesicles was determined along the whole synapse showing the typical close apposition of electron-dense presynaptic and postsynaptic membrane. We counted only vesicles without any discernible distance between vesicle and AZ membrane.

Electrophysiology. Two-electrode voltage-clamp (TEVC) recordings of EPSCs were obtained at room temperature from late third-instar male Drosophila larvae (ventral longitudinal muscle 6, segments A2 and A3), essentially as previously described (Kittel et al., 2006). For the TEVC experiments with $b r p^{n u d e}, b r p^{1.3}$, and $b r p^{5.45}$ mutants, both mutants and control animals carried a copy of ok6-GAL4 to ensure comparability with a previous study (Kittel et al., 2006). The composition of the extracellular hemolymph-like saline (HL-3) was as follows (in $\mathrm{mm}$ ): $\mathrm{NaCl} 70, \mathrm{KCl} 5$, $\mathrm{MgCl}_{2} 20, \mathrm{NaHCO}_{3} 10$, trehalose 5, sucrose 115, HEPES 5, $\mathrm{CaCl}_{2} 1$ or as indicated, and $\mathrm{pH}$ adjusted to 7.4. Recordings were made from cells with an initial resting membrane potential between -50 and $-70 \mathrm{mV}$ (holding potential at $-60 \mathrm{mV}$ ) using intracellular electrodes with resistances of 10-32 M $\Omega$, filled with $3 \mathrm{M} \mathrm{KCl}$. Train stimulation protocols consisted of 100 pulses applied at $60 \mathrm{~Hz}$. The recovery was assessed by evoking APs at (in ms following the last pulse in the train): 25, 50, 100, 200, 500, 1000, $2000,5000,10,000,20,000,50,000$, and 100,000 (Wu et al., 2005). EPSCs reflect the compound response to stimulation of both motoneurons innervating muscle 6 , and care was therefore taken to ensure their stable recruitment. Infrequently observed recruitment failures were linearly interpolated. Only cells that recovered at least $70 \%$ of their initial EPSC amplitude following tetanic stimulation were included in the analysis. The recordings were analyzed with pClamp 9 (Molecular Devices), and the peak amplitude was determined as the difference between the peak value of the EPSC and the baseline value before onset of that EPSC. The steady-state EPSC amplitudes (see Fig. 3D) were evaluated for each experiment as the average of the last 25 EPSC amplitudes in the train and normalized to the average steady-state EPSC amplitude of the corresponding control for either brp or Cac RNAi experiments. The time constants of the fast and slow components of recovery (see Fig. $3 E, F$ ) were determined from monoexponential functions fitted to the average fast and slow component of recovery and weighted with the errors of the average data with Igor Pro 6.1 (Wavemetrics). The estimated errors of the fit parameters were taken from Igor Pro (which are based on the square roots of the diagonal elements of the covariance matrix). From the time constants, the SEs, and the number of experiments, statistical comparisons were performed with Student's $t$ test, and the error bars of the normalized values were calculated according to Gaussian error propagation.

Statistical analysis. The nonparametric Mann-Whitney rank sum test was used for statistical analysis if not stated otherwise. The data are reported as mean $\pm \mathrm{SE}, n$ indicates the sample number, and $p$ denotes the significance $\left({ }^{*} p<0.05,{ }^{* *} p<0.01\right.$, and $\left.{ }^{* * *} p<0.001\right)$.

\section{Results}

\section{Impaired vesicle tethering at AZs of a Bruchpilot mutant} (brp ${ }^{\text {nude }}$ ) lacking the last $17 \mathrm{C}$-terminal amino acids

In a chemical [ethyl methyl sulfonate (EMS)] mutagenesis screen, a novel brp allele was identified with a premature STOP codon at amino acid position $1724\left(b r p^{5.38}\right.$, hereafter $\left.b r p^{\text {nude }}\right)$ of 1740 aa. While in the previously published mutants $b r p^{5.45}$ and $b r p^{1.3}$ a significant proportion of the C-terminal amino acids are absent (50\% and 30\%, respectively; STOP codons at position 867 or 1390) (Fouquet et al., 2009), brp ${ }^{\text {nude }}$ lacks only the last $1 \%$ (Fig. $1 A, C)$. Nevertheless, all three mutants showed significantly reduced survival rates and motor abilities as adult flies (Fig. $1 B$ ).

At neuromuscular junctions of $b r p^{\text {nude }}$, the reactivities of antibodies directed against the C-term (BRP ${ }^{\mathrm{Nc} 82}$; Wagh et al., 2006) and the $\mathrm{N}$-term $\left(\mathrm{BRP}^{\mathrm{N} \text {-term }}\right.$ ) (Fouquet et al., 2009) of BRP are still present (Fig. 1D), and the number of AZs per junction is normal (quantified as $\mathrm{BRP}^{\mathrm{Nc} 82}$ spots per junction; $553 \pm 27$ and $518 \pm 28$ for control and $b r p^{\text {nude }} ; n_{\text {junction }}=6$ and $\left.6 ; p=0.24\right)$. Furthermore, high-resolution light microscopic images (STED microscopy) (Hell, 2007) of BRP ${ }^{\mathrm{Nc82}}$ acquired simultaneously with confocal images of $\mathrm{BRP}^{\mathrm{N} \text {-term }}$ appeared unchanged compared to controls. Especially, the previously described ring-like distribution of Nc82 reactivity (Kittel et al., 2006) is preserved at $b r p^{\text {nude }}$ synapses (Fig. 1D). The normal distribution of $\mathrm{C}$ - and N-terminal signals at $b r p^{n u d e} \mathrm{AZs}$ indicates that the missing $1 \%$ of BRP does not alter the overall structure of the cytomatrix. In fact, an electron microscopic analysis of the ultrastructure of $b r p^{\text {nude }}$ synapses revealed normal amounts of AZ cytomatrix and an ordinary height and platform length of dense bodies (T-bars) (Fig. $1 E, F)$. Furthermore, the diameter of the synaptic vesicles as well as the total number of synaptic vesicles per bouton section were unchanged in $b r p^{\text {nude }}$ (diameter: $36 \pm 1.7$ and $35 \pm 0.3 \mathrm{~nm}$ for control and $b r p^{\text {nude }}, n_{\text {vesicles }}=220$ and 243 (10 larvae each), respectively; $p=0.9$; number: $114 \pm 24$ and $131 \pm 7 \mathrm{SV} / \mu \mathrm{m}^{2}$ for control and $b r p^{\text {nude }}, n_{\text {bouton-section }}=6$ and 6 , respectively; $p=$ $0.2)$. Finally, the number of docked vesicles per AZ section was not significantly different between $b r p^{\text {nude }}$ and controls $(1.8 \pm 0.2$ and $1.4 \pm 0.3$ for control and $b r p^{\text {nude }}, n_{\mathrm{AZ}}=21$ and 24 , respectively; $p=0.4$ ) (see Fig. $1 G$; for definition of docked vesicles, see Materials and Methods). However, T-bars at $b r p^{\text {nude }}$ AZs were bare of vesicles (Fig. $1 E$ ). A quantification of the number of vesicles within three shells of each $50 \mathrm{~nm}$ thickness surrounding the $A Z$ revealed a significant reduction in the average number of vesicles near the T-bar at $b r p^{\text {nude }}$ synapses (Fig. 1G). These data indicate that while the basic ultrastructure of the cytomatrix is unaltered at $b r p^{n u d e} \mathrm{AZs}$, vesicle tethering is specifically impaired.

\section{Normal $\mathrm{Ca}^{2+}$ channel clustering and basal release at brp $^{\text {nude }}$ synapses}

Using STED microscopy, we tested whether $\mathrm{Ca}^{2+}$ channel clustering is affected at $b r p^{\text {nude }}$ synapses. STED images of GFP-labeled $\mathrm{Ca}^{2+}$ channels ( $\mathrm{Cac}{ }^{\mathrm{GFP}}$ ) were acquired simultaneously with confocal images of postsynaptic glutamate receptors (GluRIID) to identify the position of AZs (opposite GluRIID patches) independently of $\mathrm{Ca}^{2+}$ channel clustering (Fig. 2A). Fitting Gaussian functions to the intensity profiles of average images revealed a normal $\mathrm{Ca}^{2+}$ channel distribution at $b r p^{\text {nude }}$ synapses (Fig. 2 B). Comparable results were obtained when the Cac signals were aligned with themselves. With this method, the delocalization of $\mathrm{Ca}^{2+}$ channels in brp null mutants (Kittel et al., 2006) could clearly be resolved (supplemental Fig. S1, available at www. jneurosci.org as supplemental material). Thus, we isolated a $b r p$ allele lacking only the last $1 \%$ of $\mathrm{C}$-terminal amino acids, which severely affects vitality but not the clustering of AZ $\mathrm{Ca}^{2+}$ channels.

Next, we tested the functional consequences of defective vesicle tethering. EPSCs evoked at $0.2 \mathrm{~Hz}$ at $b r p^{n u d e}$ synapses did not differ in terms of their peak amplitude, rise time, or decay time constant from those of controls (Fig. 2C). In $b r p^{1.3}$ and $b r p^{5.45}$ mutants, with poorly clustered $\mathrm{Ca}^{2+}$ channels (Fouquet et al., $2009)$, the EPSC amplitudes were reduced to $30 \pm 8 \%(n=4)$ and $10 \pm 8 \%(n=12)$ of control amplitudes, respectively (Fig. $2 C)$. The amplitude of miniature EPSCs was not significantly affected in any of these three mutants (Fig. 2D). These data indicate that basal release $\left(\right.$ at $0.2 \mathrm{~Hz}$ ) requires $\mathrm{Ca}^{2+}$ channel clustering, but not vesicles tethered to the cytomatrix. 
A

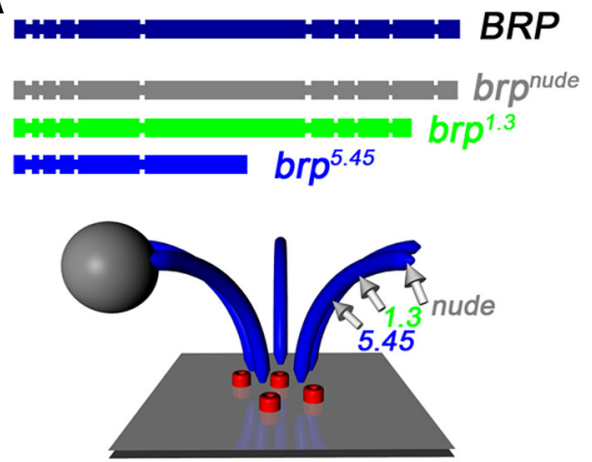

B

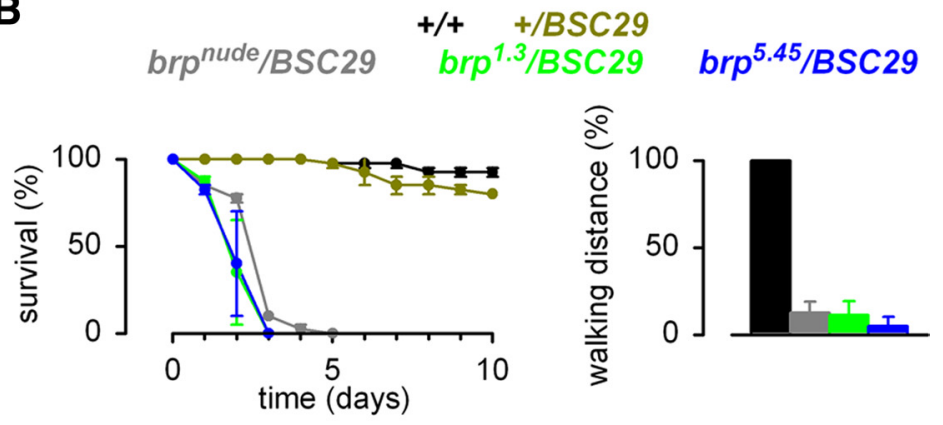

C

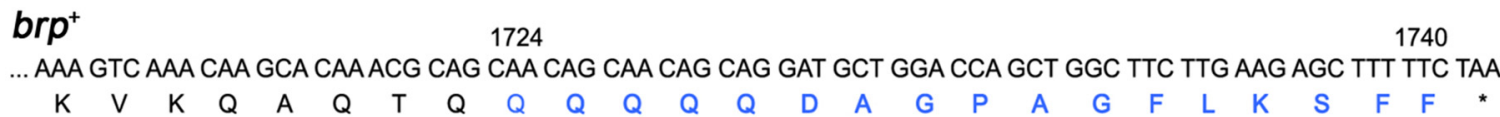

brp nude

... AAA GTC AAA CAA GCA CAAACG CAG TAA CAG CAA CAG CAG GAT GCT GGA CCA GCT GGC TTC TTG AAG AGC TTT TTC TAA $\mathrm{K}$
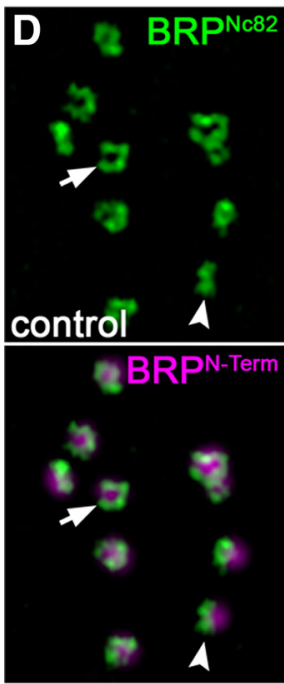
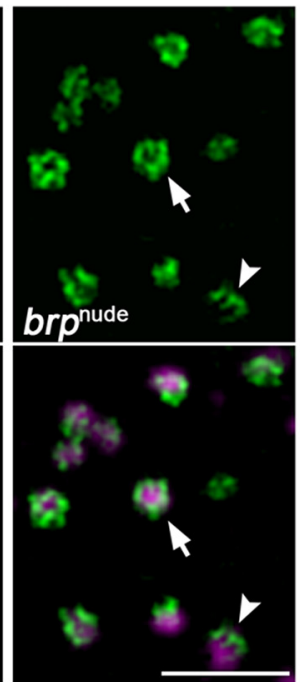
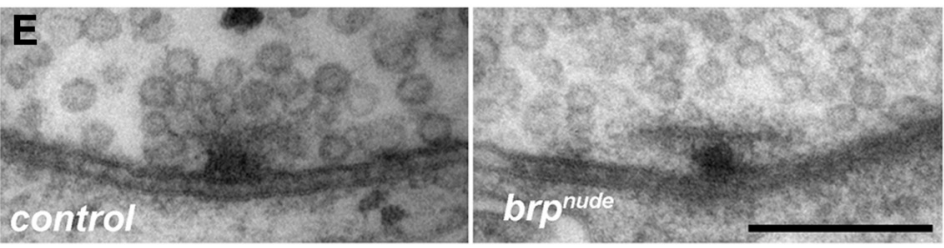

$\mathbf{F}$

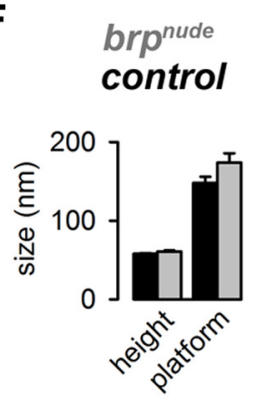

G

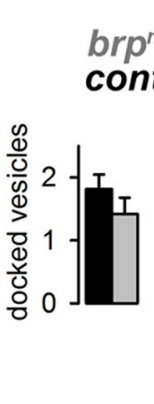

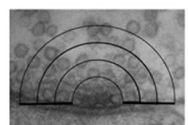

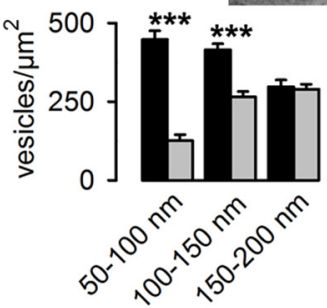

Figure 1. Impaired vesicle tethering at AZs of a bruchpilot mutant ( $\left.b r p^{\text {nude }}\right)$ lacking the last 17 C-terminal amino acids. $A$, Chemical induction of premature stop codons resulted in truncated versions of the BRP protein (BRP; dark blue; boxes indicate coiled-coil domains) of $\sim 99 \%$ (brp ${ }^{\text {nude }}$; gray), of $\sim 70 \%$ (brp ${ }^{1.3}$; green), and of $\sim 50 \%$ length (brp. ${ }^{5.45}$; blue). Below, Illustration of the BRP protein (blue) within the AZ (red: $\mathrm{Ca}^{2+}$ channels; gray: synaptic vesicle) and the corresponding positions of the truncation (arrows) (Fouquet et al., 2009). B, All three BRP mutants (brp ${ }^{\text {nude }}$, brp ${ }^{1.3}$; $b r p^{5.45}$ ) showed severely impaired survival rates and walking skills. $C$, In the brp nude allele, a single base mutation at the C-terminal position 1724 leads to a premature stop codon and a BRP protein (BRP nude) lacking the last 17 of 1740 aa. D, STED images of neuromuscular junctions stained with a (-terminal BRP antibody (green; BRP Nc82) and simultaneous confocal images of an N-terminal BRP antibody (magenta; BRP ${ }^{\mathrm{N} \text {-term }}$ ). The distribution of both antibody signals appears unaltered at $b r p^{\text {nude }}$ synapses (right) compared to controls (left). Arrows and arrowheads indicate planar and vertical AZs, respectively. $\boldsymbol{E}$, Examples of conventionally embedded AZs of control and $b r p^{\text {nude }}$. Note fewer tethered vesicles in $b r p^{\text {nude }}$. $\boldsymbol{F}$, Height and length of the platform of the electron-dense cytomatrix (T-bar) for control and brp ${ }^{\text {nude }}$. G, The number of docked vesicles per AZ section for control and $b r p^{\text {nude }}$ ( $n=22$ and $25 \mathrm{AZs}$, respectively) and the number of vesicles found within three shells (see inset) each of $50 \mathrm{~nm}$ thickness surrounding the AZ ( $n=20$ and 31 AZs, respectively). Scale bars: $\boldsymbol{D}, 1 \mu \mathrm{m} ; \boldsymbol{E}, 200 \mathrm{~nm}$.

Tethering vesicles to the active zone dense body prevents synaptic depression

To investigate the impact of vesicle tethering on short-term plasticity, we analyzed synaptic transmission during paired-pulse stimulation (Fig. 3A). Strikingly, the amplitude of the second EPSC was significantly reduced at short interpulse intervals of 10 and $30 \mathrm{~ms}$ (Fig. 3B). This depression decayed with a time constant of $19 \mathrm{~ms}$. Next, synaptic transmission was analyzed during a train of 100 stimuli at $60 \mathrm{~Hz}$ (Fig. 3C) (Hallermann et al., 2010). The depression during the train was stronger in $b r p^{\text {nude }}$ as quantified by a reduction in the steady-state EPSC amplitude at the end of the train to $35 \pm 3 \%$ compared to $52 \pm 5 \%$ in controls $(n=21$ and 20 for control and $b r p^{\text {nude }}$ mutants, respectively) (Fig. $3 C, D$ ). Consistent with the altered paired-pulse ratio, the amplitude of the second EPSC in the train was already significantly reduced to $80 \pm 10 \%$ at $b r p^{n u d e}$ synapses $(n=21$ and 20$)$.

If sustained release is impaired in $b r p^{n u d e}$ mutants because fewer vesicles are tethered to the cytomatrix, then sustained release in $b r p^{1.3}$ and $b r p^{5.45}$ mutants, both of which show severely impaired cytomatrices (Fouquet et al., 2009), should be im- 


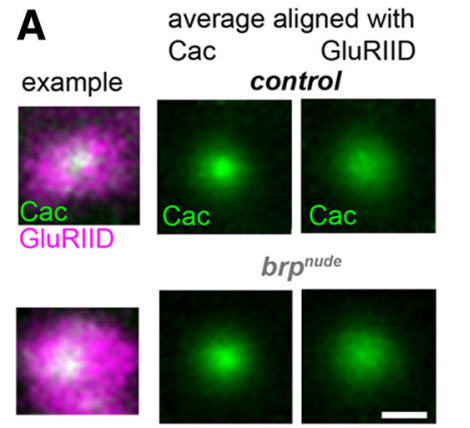

B
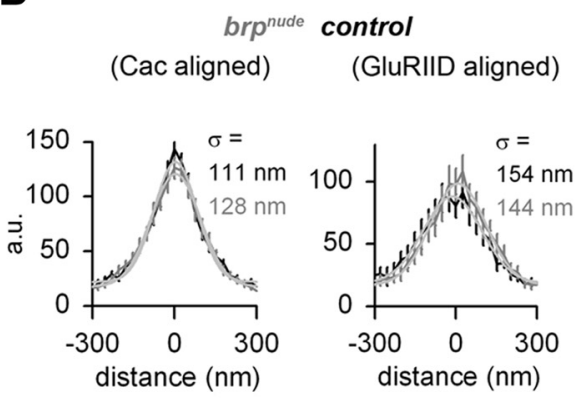

C
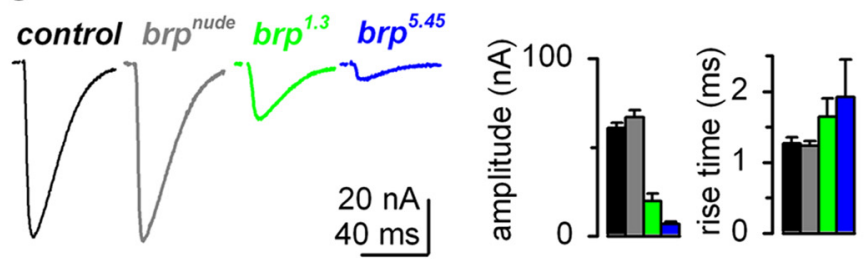

D

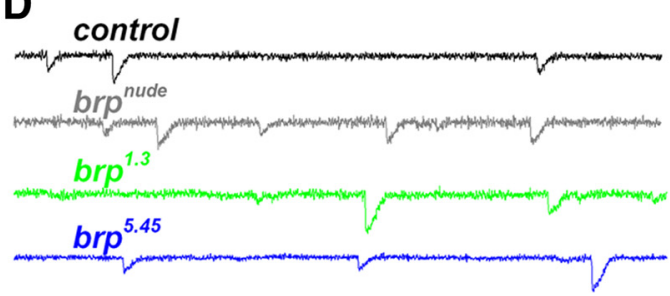

$2 \mathrm{nA}$

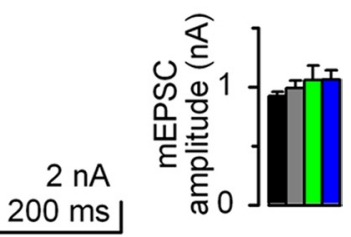

Figure 2. Normal $\mathrm{Ca}^{2+}$ channel clustering and basal release at $b r p^{\text {nude }}$ synapses. $A$, GluRIID labels (magenta, confocal) were used to quantify the $\mathrm{Ca}^{2+}$ channel ( $\mathrm{Cac}{ }^{\text {GFP }}$, green, STED) clusters in control animals (top row) and brp ${ }^{\text {nude }}$ mutants (below). The Cac signals at synapses that appeared ideally planar ( $n=16$ each) were averaged after alignment with the postsynaptic GluRIID signal (right panels) or themselves (center panels). While the example images were scaled up individually, all four averages were scaled up with the same factor. $\boldsymbol{B}$, Intensity profiles of the average Cac images in black for controls and in gray for brp ${ }^{\text {nude }}$ mutants with SE bars and the corresponding Gaussian fits (standard deviations given by $\sigma$ ) in gray. C, Examples of EPSCs elicited at $0.2 \mathrm{~Hz}$ in control (black), $b r p^{\text {nude }}$ (gray), $b r p^{1.3}$ (green), and $b r p^{5.45}$ mutants (blue; average of 10 each) in $1.0 \mathrm{~mm} \mathrm{Ca}{ }^{2+}$. The average peak EPSC amplitudes and rise and decay kinetics were normal in $b r p^{\text {nude }}$ mutants. In $b r p^{1.3}$ and $b r p^{5.45}$ mutants EPSC amplitudes were reduced and rise times increased $\left(n=22,18,4\right.$, and 12 for control, $b r p^{\text {nude }}$, $b r p^{1.3}$, and $b r p^{5.45}$, respectively). $\boldsymbol{D}$, Example traces of $\mathrm{mEPSC}$ of control (black), $b r p^{\text {nude }}$ (gray), $b r p^{1.3}$ (green), and $b r p^{5.45}$ mutants (blue). The average $\mathrm{mEPSC}$ amplitudes were normal in $b r p^{\text {nude }}, \operatorname{brp}^{1.3}$, and $b r p^{5.45}$ mutants $\left(n=6,6,4\right.$, and 6 for control, brp ${ }^{\text {nude }}$, $b r p^{1.3}$, and $b r p^{5.45}$, respectively). Scale bar in $\boldsymbol{A}, 250 \mathrm{~nm}$.

paired, too. Indeed, investigations of sustained release in $b r p^{1.3}$ and $b r p^{5.45}$ mutants revealed stronger synaptic depression than in controls (supplemental Fig. S2 A, C, available at www.jneurosci.org as supplemental material). To analyze whether the stronger depression in $b r p^{1.3}$ and $b r p^{5.45}$ mutants is simply the consequence of impaired $\mathrm{Ca}^{2+}$ channel clustering, we sought to compensate ("rescue") the reduced $\mathrm{Ca}^{2+}$ channel density by increasing the extracellular $\mathrm{Ca}^{2+}$ concentration. In high $\mathrm{Ca}^{2+}\left(2 \mathrm{~mm}\right.$ and $2.5 \mathrm{~mm}$ for $b r p^{1.3}$ and $b r p^{5.45}$ mutants, respectively), their EPSC amplitudes were comparable to those of controls in $1 \mathrm{~mm} \mathrm{Ca}^{2+}$. However, depression during sustained release was still pronounced (Fig. 3D; supplemental Fig. $\mathrm{S} 2 B, D$, available at www.jneurosci.org as supplemental material).

Since vesicle recruitment critically depends on the intracellular spatiotemporal $\mathrm{Ca}^{2+}$ dynamics (Neher and Sakaba, 2008), we performed control experiments by reducing the density of $\mathrm{Ca}^{2+}$ channels genetically and tested whether subsequent elevations of extracellular $\mathrm{Ca}^{2+}$ could rescue sustained release. To this end, transgene-mediated RNAi directed against the $\alpha 1$ subunit of the Drosophila $\mathrm{Ca}^{2+}$ channel (Cac) was performed, resulting in a reduction of EPSC amplitudes by $\sim 50 \%$. Extracellular $\mathrm{Ca}^{2+}$ was then elevated to $1.5 \mathrm{~mm}$ to obtain control EPSC amplitudes. However, in

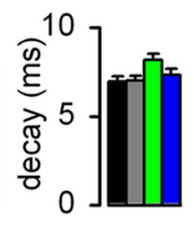

contrast to $b r p^{1.3}$ and $b r p^{5.45}$ mutants, sustained release was unaltered by Cac RNAi (Fig. 3D; supplemental Fig. S2 E, available at www.jneurosci.org as supplemental material).

Next, we addressed whether the enhanced depression during sustained highfrequency transmission at $b r p^{\text {nude }}$ synapses is accompanied by alterations in the kinetics of recovery from depression. Therefore, the recovery from synaptic depression after a train was investigated with test stimuli of increasing intervals following the train (Fig. 3E, F) (Wu et al., 2005; Hallermann et al., 2010). A biphasic recovery with time constants of $\tau_{1}=50$ $\mathrm{ms}$ and $\tau_{2}=6.1 \mathrm{~s}$ was found in controls. In $b r p^{\text {nude }}$ mutants, the first component was slower (114 ms), while the second component was unaltered (5.6 s) (Fig. 3E,F). Consistently, the first component of recovery was also slower in $b r p^{1.3}$ and $b r p^{5.45}$ mutants with $\mathrm{Ca}^{2+}$ concentrations that rescued the basal EPSC amplitude. In contrast to the normal second component of recovery at $b r p^{\text {nude }}$ synapses, the second component of recovery in $b r p^{1.3}$ and $b r p^{5.45}$ mutants was also significantly slower (Fig. 3 E, F; supplemental Fig. S2, available at www.jneurosci.org as supplemental material). Finally, in experiments with Cac RNAi, which serve as a control for the approach of elevating $\mathrm{Ca}^{2+}$, both components were normal (Fig. 3 E, F; supplemental Fig. S2, available at www. jneurosci.org as supplemental material). In summary, these data indicate that the basal EPSC amplitude (cf. Fig. 2) as well as the second component of recovery rely on adequate $\mathrm{Ca}^{2+}$ channel clustering (impaired in $b r p^{1.3}$ and $b r p^{5.45}$ ) and that highfrequency sustained release as well as the first component of recovery also rely on proper vesicle tethering at the AZ (selectively impaired in $b r p^{\text {nude }}$ ).

\section{Discussion}

To our knowledge, the specific impairment of vesicle tethering reported here delivers the first direct demonstration that efficient sustained release relies on the ability of the AZ to tether vesicles. While the overall AZ structure, including the distribution of $\mathrm{Ca}^{2+}$ channels, was unaffected, the impairment of vesicle tethering provoked pronounced synaptic depression and a slowed first component of recovery.

The C-terminal half of BRP consists of $\sim 1000$ aa of essentially contiguous coiled-coil sequence (Wagh et al., 2006), reminiscent of Golgi/ER-resident tethering factors such as, e.g., GM130 (Lupashin and Sztul, 2005). These coiled-coils typically form rod-like structures, where 100 aa residues extend $\sim 15 \mathrm{~nm}$ when dimerized, and proteins such as Usolp (Yamakawa et al., 1996) extend over $150 \mathrm{~nm}$ (Lupashin and Sztul, 2005). These rod-like proteins are believed to act before SNARE protein assembly by forming contacts between membranes at a distance, thereby increasing the specificity or efficiency of the initial at- 

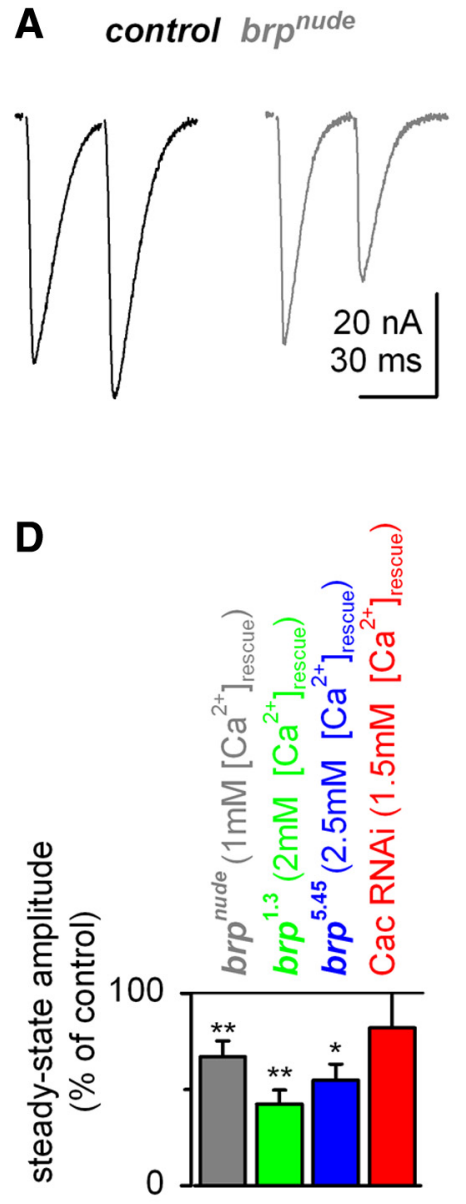

B

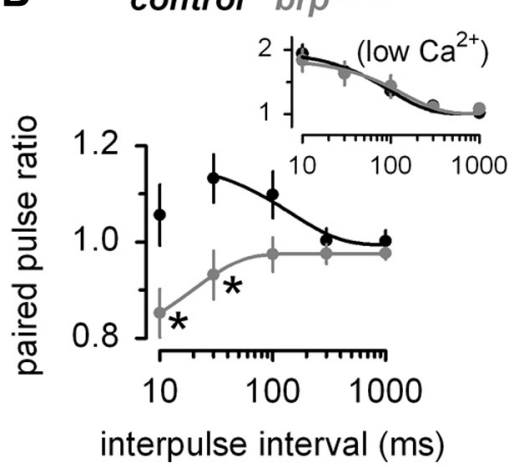

E

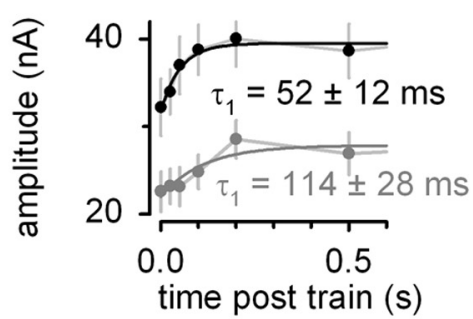

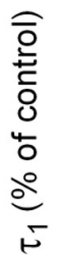

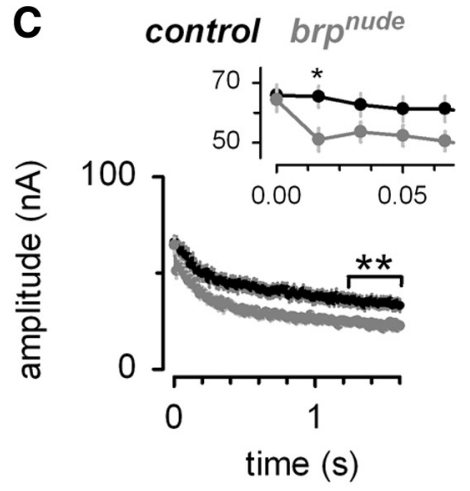

$\mathbf{F}$
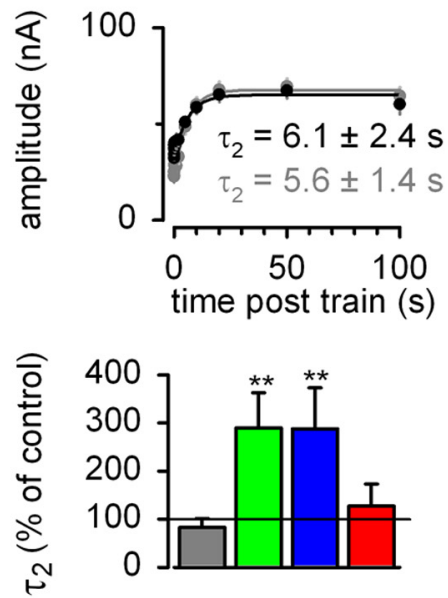

Figure 3. Tethering vesicles to the AZ dense body prevents synaptic depression. $A$, Examples of EPSCs elicited by a paired-pulse protocol with $30 \mathrm{~ms}$ interpulse interval in control (black) and $b r p^{\text {nude }}$ (gray). B, Average paired-pulse ratios plotted versus interpulse interval in $1.0 \mathrm{~mm} \mathrm{Ca}^{2+}$ for control (black, $n=12$ ) and brp ${ }^{\text {nude }}$ (gray, $n=9$; asterisk indicates significant differences) superimposed with exponential fits (time constants are indicated). Inset, $0.6 \mathrm{~mm} \mathrm{Ca}^{2+}$. C, Average peak EPSC amplitudes during the $60 \mathrm{~Hz}$ train for control (black, $\left.n=21\right)$ and brp ${ }^{\text {nude }}$ ( $g$ ray, $n=$ 20). Inset, Average amplitudes of the first 5 EPSCs of the train. The steady-state amplitude (average of EPSC amplitudes marked by the bracket) was significantly reduced in brp ${ }^{\text {nude }}$ mutants compared to controls. D, Steady-state amplitudes normalized to the steady-state amplitude of the respective control, for brp ${ }^{\text {nude }}$ (gray), brp ${ }^{1.3}$ (green), brp ${ }^{5.45}$ (blue), and Cac RNAi synapses (red) in extracellular $\mathrm{Ca}^{2+}$ concentrations that lead to normal ("rescued") basal EPSC amplitudes ([Ca $\left.{ }^{2+}\right]_{\text {rescue }}$ ) (see supplemental Fig. S2, available at www.jneurosci.org as supplemental material). $\boldsymbol{E}$, Average peak EPSC amplitudes during the first rapid component of recovery after the train $\left(\tau_{1}\right)$ for control (black, $\left.n=21\right)$ and brp ${ }^{\text {nude }}$ (gray, $\left.n=20\right)$ fitted exponentially (lines). Time constants with SEs are indicated. Bottom, The time constants of the first component of recovery normalized to the corresponding time constant of the control (color code as in D). $F$, Corresponding average peak EPSC amplitudes during the second slower component of recovery after the train $\left(\tau_{2}\right)$ with corresponding average data.

tachment of vesicles (tethering) (Guo et al., 2000). We have provided morphological and functional evidence that BRP filaments tether vesicles, and thus further mechanistic comparisons between AZ and Golgi/ER trafficking, e.g., concerning the role of small GTPases, might well be informative.

The C-terminal half of BRP is very highly conserved in insects but not elsewhere (Wagh et al., 2006). Interestingly, the Drosophila genome does not appear to encode homologs of the vertebrate AZ components Piccolo and Bassoon (Wagh et al., 2006), which are key regulators of the vertebrate cytomatrix (Khimich et al., 2005). At central vertebrate synapses, CAST and Bassoon immunoreactivities (closer and further from the AZ membrane, respectively) were recently found to be associated with filaments that may connect vesicles to the AZ (Siksou et al., 2007). It is tempting to speculate that at AZs of central vertebrate synapses, CAST associates with coiled-coil domain proteins, such as bassoon, to perform the dual functions of $\mathrm{Ca}^{2+}$ channel clustering and vesicle tethering executed by the $\mathrm{N}$-terminal and the C-terminal domains of BRP, respectively.

How synapses manage to repetitively release transmitter with high precision is intensely investigated. Vesicles tethered to electron-dense bodies may represent a reservoir of vesicles required for sustained release (Zhai and Bellen, 2004). Consistent with this hypothesis, synaptic stimulation provokes depletion of vesicles tethered at dense bodies (LoGiudice et al., 2008; Jackman et al., 2009). While the supply of vesicles appears rate limiting during the train and the first component of recovery (Saviane and Silver, 2006), the maturation of vesicles closer to $\mathrm{Ca}^{2+}$ channels appears rate limiting during the second component of recovery [cf. $b r p^{1.3}$ and $b r p^{5.45}$ (Kittel et al., 2006; Fouquet et al., 2009)]. One may argue that the rapid component of depression observed at $b r p^{\text {nude }}$ synapses (Fig. $3 A, B$ ) could be partially attributed to fewer docked vesicles (though not significantly; cf. Fig. $1 G$ ) with a higher initial release probability. However, a functional estimation of the number of readily releasable vesicles using backextrapolation from the cumulative EPSC amplitudes in the trains (Schneggenburger et al., 1999) revealed similar numbers of readily releasable vesicles in $b r p^{\text {nude }}$ and controls (supplemental Fig. S3, available at www.jneurosci.org as supplemental material). Finally, we would like to point out that the C-term of BRP could be involved in endocytotic mechanisms, which have been shown to be crucial for sustained release (Koenig et al., 1998; 
Kawasaki et al., 2000; Dickman et al., 2005; Hosoi et al., 2009; Yao et al., 2009). Novel techniques have begun to address the spatial organization of local vesicle reuse within active zones (Zhang et al., 2009). It will have to be clarified via which routes vesicles move within active zones and in which direction Bruchpilot steers their translocation.

\section{References}

Atwood HL, Karunanithi S (2002) Diversification of synaptic strength: presynaptic elements. Nat Rev Neurosci 3:497-516.

Dickman DK, Horne JA, Meinertzhagen IA, Schwarz TL (2005) A slowed classical pathway rather than kiss-and-run mediates endocytosis at synapses lacking synaptojanin and endophilin. Cell 123:521-533.

Dietzl G, Chen D, Schnorrer F, Su KC, Barinova Y, Fellner M, Gasser B, Kinsey K, Oppel S, Scheiblauer S, Couto A, Marra V, Keleman K, Dickson BJ (2007) A genome-wide transgenic RNAi library for conditional gene inactivation in Drosophila. Nature 448:151-156.

Fouquet W, Owald D, Wichmann C, Mertel S, Depner H, Dyba M, Hallermann S, Kittel RJ, Eimer S, Sigrist SJ (2009) Maturation of active zone assembly by Drosophila Bruchpilot. J Cell Biol 186:129-145.

Guo W, Sacher M, Barrowman J, Ferro-Novick S, Novick P (2000) Protein complexes in transport vesicle targeting. Trends Cell Biol 10:251-255.

Hallermann S, Heckmann M, Kittel RJ (2010) Mechanisms of short-term plasticity at neuromuscular active zones of Drosophila. HFSP J 4:72-84.

Hell SW (2007) Far-field optical nanoscopy. Science 316:1153-1158.

Hosoi N, Holt M, Sakaba T (2009) Calcium dependence of exo- and endocytotic coupling at a glutamatergic synapse. Neuron 63:216-229.

Jackman SL, Choi SY, Thoreson WB, Rabl K, Bartoletti TM, Kramer RH (2009) Role of the synaptic ribbon in transmitting the cone light response. Nat Neurosci 12:303-310.

Kawasaki F, Hazen M, Ordway RW (2000) Fast synaptic fatigue in shibire mutants reveals a rapid requirement for dynamin in synaptic vesicle membrane trafficking. Nat Neurosci 3:859-860.

Khimich D, Nouvian R, Pujol R, Tom Dieck S, Egner A, Gundelfinger ED, Moser T (2005) Hair cell synaptic ribbons are essential for synchronous auditory signalling. Nature 434:889-894.

Kidokoro Y, Kuromi H, Delgado R, Maureira C, Oliva C, Labarca P (2004) Synaptic vesicle pools and plasticity of synaptic transmission at the Drosophila synapse. Brain Res Brain Res Rev 47:18-32.

Kittel RJ, Wichmann C, Rasse TM, Fouquet W, Schmidt M, Schmid A, Wagh DA, Pawlu C, Kellner RR, Willig KI, Hell SW, Buchner E, Heckmann M,
Sigrist SJ (2006) Bruchpilot promotes active zone assembly, $\mathrm{Ca}^{2+}$ channel clustering, and vesicle release. Science 312:1051-1054.

Koenig JH, Yamaoka K, Ikeda K (1998) Omega images at the active zone may be endocytotic rather than exocytotic: implications for the vesicle hypothesis of transmitter release. Proc Natl Acad Sci U S A 95:12677-12682.

LoGiudice L, Sterling P, Matthews G (2008) Mobility and turnover of vesicles at the synaptic ribbon. J Neurosci 28:3150-3158.

Lupashin V, Sztul E (2005) Golgi tethering factors. Biochim Biophys Acta 1744:325-339.

Neher E, Sakaba T (2008) Multiple roles of calcium ions in the regulation of neurotransmitter release. Neuron 59:861-872.

Saviane C, Silver RA (2006) Fast vesicle reloading and a large pool sustain high bandwidth transmission at a central synapse. Nature 439:983-987.

Schneggenburger R, Meyer AC, Neher E (1999) Released fraction and total size of a pool of immediately available transmitter quanta at a calyx synapse. Neuron 23:399-409.

Siksou L, Rostaing P, Lechaire JP, Boudier T, Ohtsuka T, Fejtová A, Kao HT, Greengard P, Gundelfinger ED, Triller A, Marty S (2007) Threedimensional architecture of presynaptic terminal cytomatrix. J Neurosci 27:6868-6877.

Wagh DA, Rasse TM, Asan E, Hofbauer A, Schwenkert I, Dürrbeck H, Buchner S, Dabauvalle MC, Schmidt M, Qin G, Wichmann C, Kittel R, Sigrist SJ, Buchner E (2006) Bruchpilot, a protein with homology to ELKS/ CAST, is required for structural integrity and function of synaptic active zones in Drosophila. Neuron 49:833-844.

Wu Y, Kawasaki F, Ordway RW (2005) Properties of short-term synaptic depression at larval neuromuscular synapses in wild-type and temperature-sensitive paralytic mutants of Drosophila. J Neurophysiol 93:2396-2405.

Yamakawa H, Seog DH, Yoda K, Yamasaki M, Wakabayashi T (1996) Usol protein is a dimer with two globular heads and a long coiled-coil tail. J Struct Biol 116:356-365.

Yao CK, Lin YQ, Ly CV, Ohyama T, Haueter CM, Moiseenkova-Bell VY, Wensel TG, Bellen HJ (2009) A synaptic vesicle-associated $\mathrm{Ca}^{2+}$ channel promotes endocytosis and couples exocytosis to endocytosis. Cell 138:947-960.

Zhai RG, Bellen HJ (2004) The architecture of the active zone in the presynaptic nerve terminal. Physiology (Bethesda) 19:262-270.

Zhang Q, Li Y, Tsien RW (2009) The dynamic control of kiss-and-run and vesicular reuse probed with single nanoparticles. Science 323:1448-1453.

Zucker RS, Regehr WG (2002) Short-term synaptic plasticity. Annu Rev Physiol 64:355-405. 